\title{
Cooling simulation and experimental benchmarking for an rf-based electron cooler
}

\author{
H. Zhao๑, ${ }^{*}$ M. Blaskiewicz, A. V. Fedotov®, W. Fischer, X. Gu๑, D. Kayran, \\ J. Kewisch, C. Liu, S. Seletskiy, V. Schoefer, and P. Thieberger® \\ Brookhaven National Laboratory, Upton, New York 11973, USA
}

(Received 30 March 2020; accepted 1 June 2020; published 1 July 2020)

\begin{abstract}
The Low Energy Relativistic Heavy Ion Collider (RHIC) Electron Cooler (LEReC) is the world's first electron cooler using rf-accelerated electron bunches. Recently, the cooling of gold ion beams in RHIC by 1.6 and $2.0 \mathrm{MeV}$ electrons was successfully achieved. Along with the velocity spread and alignment of the electron beam, the space-charge force between ions and electrons also plays an important role in the cooling process. In order to investigate the cooling dynamics with bunched electron beams and to provide guidance for the LEReC operation, a simulation code was developed, which includes nonmagnetized cooling, intrabeam scattering, and the space-charge effect. In this paper, we present and discuss the simulation results, showing how various effects influence the cooling process as well as provide experimental benchmarking of the simulations.
\end{abstract}

DOI: 10.1103/PhysRevAccelBeams.23.074201

\section{INTRODUCTION}

In the past few decades, the electron cooling method [1] has been widely applied in several low-energy proton and ion storage rings [2-3]. It is a powerful method to shrink the size and momentum spread of the stored beams for beam accumulation and high-precision experiments [4-7]. Up to now, all electron coolers built around the world were based on dc electron beams, which are accelerated by electrostatic fields. The highest-energy electron cooling system with 4.3 MeV electrons has been successfully constructed and operated at Fermi National Accelerator Laboratory [8]. Because of the limitation of the high voltage and the beam power, it is challenging to build a higher-energy dc electron cooler. However, high-energy electron coolers are needed for future facilities, such as the Electron Ion Collider [9-10], which requires at least tens of $\mathrm{MeV}$ electron beams for cooling. rf-accelerated bunched electron cooling is one of the most promising approaches for high-energy electron cooling.

The Low Energy Relativistic Heavy Ion Collider Electron Cooler (LEReC) at Brookhaven National Laboratory is the world's first electron cooler using rfaccelerated electron bunches [11]. The ultimate goal for LEReC is to increase the luminosities for two (3.85 and

\footnotetext{
*hezhao@bnl.gov

Published by the American Physical Society under the terms of the Creative Commons Attribution 4.0 International license. Further distribution of this work must maintain attribution to the author(s) and the published article's title, journal citation, and DOI.
}

$4.59 \mathrm{GeV} / \mathrm{u}$ ) of the five beam energies of the beam energy scan phase-II (BES-II) physics program in RHIC [12]. Recently, the cooling of gold beams in RHIC by 1.6 and 2.0 MeV electrons was successfully achieved [13-14]. LEReC not only demonstrated rf-accelerated electron cooling, but also provided experience and experimental data for future research on high-energy electron coolers.

There are several challenges that had to be overcome to demonstrate cooling with the LEReC approach. First, since there is no magnetic field in the cooling regions, the cooling performance is very sensitive to both the transverse and the longitudinal electron beam velocity spreads. In addition to the space-charge (SC) effect of the electron beam itself, the velocity spread of the electron beam is also affected by the ion beam, which makes it complicated to control the electron beam quality. Second, when passing through the cooling section (CS), the ion beam sees the SC kick from the electron bunches. Because of the synchrobetatron resonance driven by the SC force of the electrons [15], the intensity and the length of electron bunches need to be carefully chosen, especially for the beam at low energy. Finally, very good alignment between the ion and electron beams is required, because a small position offset or a small angle between the two beams has a significant influence on the cooling rate. The velocity of the electron beam should also be well matched with the ion beam [16].

In order to investigate the cooling dynamics in detail in LEReC, a simulation code named TRACKIT was developed, in which several effects relevant to the cooling approach with a bunched electron beam are included [17]. In particular, the SC effect is introduced for the first time in a cooling simulation. In this paper, we present and 


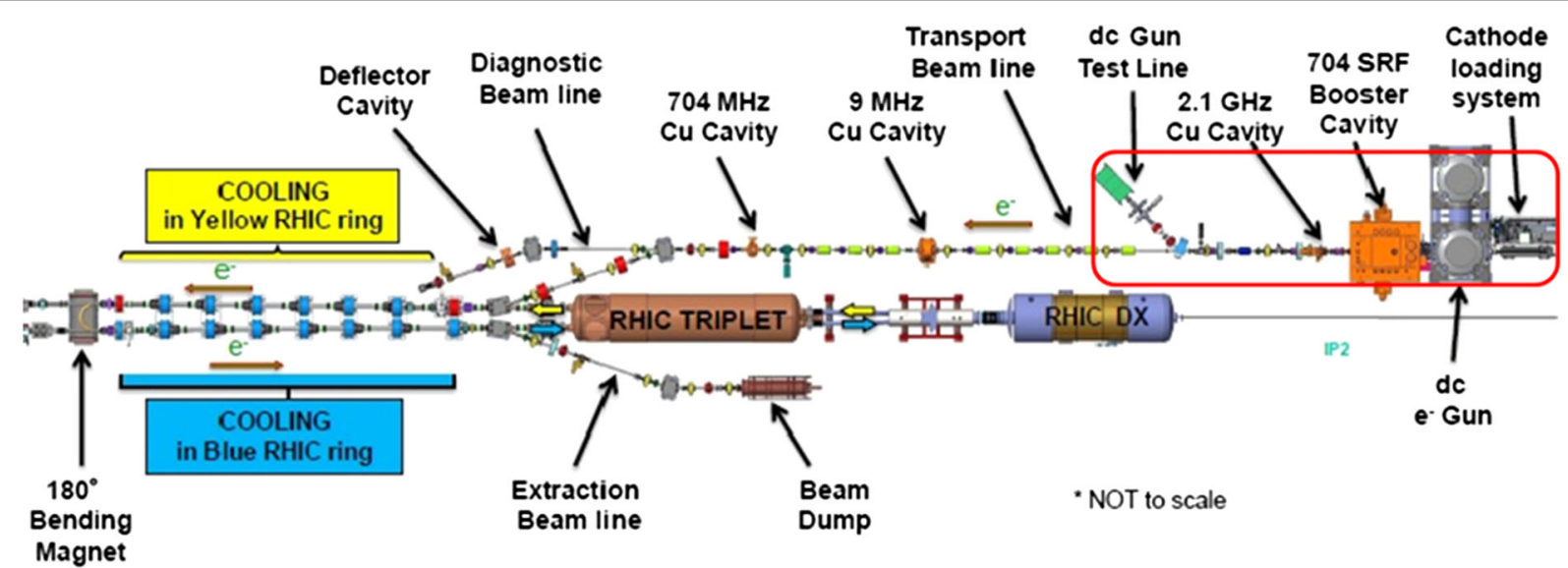

FIG. 1. Layout of the LEReC accelerator.

discuss the simulation results, showing the influence of various effects on the cooling process. The experimental benchmarking of the simulation code is also provided.

The paper is organized as follows. In Sec. II, the LEReC accelerator is introduced. In Sec. III, the physical models in the simulation code are introduced, and the benchmarking results are presented. In Sec. IV, the measured and simulated cooling processes are presented, and the effects that can influence the cooling rate are discussed. Finally, a summary is given in Sec. V.

\section{LEReC ACCELERATOR}

The layout of LEReC is shown in Fig. 1. The electron beam is generated from the photocathode, which is installed in the dc gun with the design voltage of $400 \mathrm{kV}$ [18]. A photogun with a fiber laser produces electron bunches at a repetition rate of $704 \mathrm{MHz}$ in macrobunch trains at the repetition rate of $\sim 9 \mathrm{MHz}$, which is the same as the repetition rate of the ion bunches in RHIC. Then, the electron beam is accelerated to the desired energy by the $704 \mathrm{MHz}$ superconducting radio frequency (SRF) cavity [19]. In order to prevent the degradation of the beam energy spread and emittance due to the SC effect, an energy chirp is introduced in the $704 \mathrm{MHz}$ SRF cavity to stretch the bunches. After acceleration, a series of subsequent warm cavities are applied to control the energy spread and the bunch length of the electron beam: A $2.1 \mathrm{GHz}$ cavity is used to compensate the nonlinear energy spread introduced by the SRF cavity. Another $704 \mathrm{MHz}$ cavity is used to remove the energy chirp after stretching, and an additional $9 \mathrm{MHz}$ cavity is used to compensate the beam loading effect within each bunch train. In addition, there are several solenoids along the transport beam line to control the beam size and to reduce additional emittance growth due to the SC effect. After that, the electron beam is merged into the two CSs in yellow and blue rings of RHIC. The two CSs are connected by a $180^{\circ}$ bending magnet so that the electron beam can cool the ion beam in both rings at the same time. Several solenoids are also installed in the CSs to control the electron beam envelope. After interacting with ions in the two CSs, the electron beam is finally extracted to the beam dump. Correctors, beam position monitors (BPMs), and other beam diagnosis instruments are installed along the electron beam trajectory. More details can be found in Ref. [11].

The time structures of the ion $\left({ }^{197} \mathrm{Au}^{79+}\right)$ and electron beams during cooling in RHIC are shown in Fig. 2. Each ion bunch encounters 30 electron bunches, and each electron bunch has a uniform longitudinal distribution. The LEReC cooling process is nonmagnetized, i.e., without a magnetic field from the gun to the CS. The solenoids along the trajectory are used to correct the envelope of the electron beam, and their influence on cooling performance is so weak that it can be ignored. Since no continuous longitudinal magnetic field is used in the CS, it is very important to control the beam velocity spread in all three dimensions that directly determine the cooling rate. The requirement for the rms emittance and rms momentum

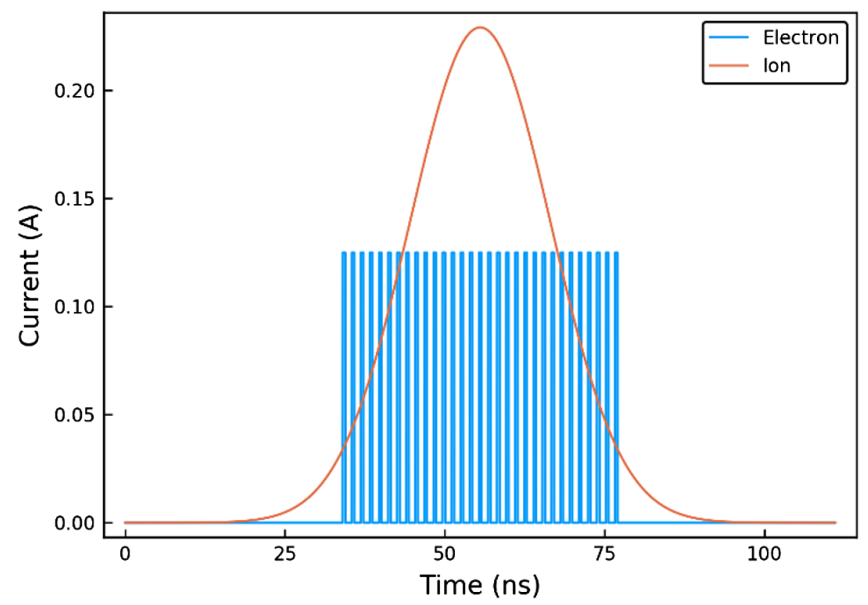

FIG. 2. Time structure of the ion and electron beam. Electrons, $50 \mathrm{pC}$ per bunch and the full bunch length of $0.4 \mathrm{~ns}$ (uniform); ions, $6.3 \mathrm{nC}$ per bunch and rms bunch length of $12 \mathrm{~ns}$. 
spread of the electron beam are $\epsilon_{x / y} \approx 0.5 \mu \mathrm{m}\left(\sigma_{\theta}=150 \mu \mathrm{rad}\right)$ and $d p / p \approx 5 \times 10^{-4}$, respectively, which were chosen to be close to the rms velocity spreads of the ion beam [11].

\section{PHYSICAL MODELS, SIMULATION, AND BENCHMARKING}

In order to obtain effective cooling in LEReC, a steady electron beam with small velocity spread is required. However, due to the SC effect, the electron beam distribution in phase space will change along the $\mathrm{CS}$ and will also be disturbed by the ion beam. This makes it difficult to optimize the electron beam for the maximum cooling rate. Moreover, the ion beam will suffer from the synchrobetatron resonance driven by the electron bunches, which will result in emittance growth of the ion beam or even cause beam loss. In general, cooling depends not only on the rms velocity spreads and density of the electron beam, but also on the distribution and intensity of the ion beam. A simulation code which includes the described effects was required to study the cooling dynamics for bunched electron beam, as well as to provide guidance for the operation.

The multiparticle tracking code TRACKIT is based on a simple one-turn map and a thin lens treatment of the electron-ion interaction. The one-turn map on each particle in the code is defined by the betatron and synchrotron tunes, coupling, chromaticities, tune spread, and sine wave rf. In this section, we introduce the physical models in the simulation, such as the cooling force, intrabeam scattering (IBS) effect, and the SC effect. These models were benchmarked with the BETACOOL code [20] and with the experimental data.

\section{A. Cooling force and IBS}

In the absence of a magnetic field, the friction force acting on the ion in electron gas is given in the particle reference frame by [21]

$\vec{F}=-4 \pi n_{e} m_{e} r_{e}^{2} Z^{2} c^{4} \int L_{c} \frac{\vec{V}_{i}-\vec{V}_{e}}{\left|\vec{V}_{i}-\vec{V}_{e}\right|^{3}} f\left(V_{e}\right) d^{3} V_{e}$,

where $n_{e}=n_{e}(x, y, z)$ is the local number density of the electron bunch, $r_{e}$ is the classical electron radius, $\vec{V}_{i}$ and $\vec{V}_{e}$ are ion and electron velocities, respectively, $f\left(\vec{V}_{e}\right)$ is the velocity distribution of the electron bunch, and $L_{c}$ is the Coulomb logarithm. The beam density and velocities are all in the frame comoving with the ion beam. Assuming that the electron beam velocity satisfies Gaussian distribution in three dimensions, the integral in Eq. (1) has the same form as the elliptical potential formulas in Ref. [22], and it can be simplified to

$$
\begin{aligned}
& F_{x, y, z}\left(V_{i x}, V_{i y}, V_{i z}\right) \\
& =K \int_{0}^{\infty} \frac{V_{i x, i y, i z}}{\sqrt{\left(\sigma_{V_{e x}}^{2}+\lambda\right)\left(\sigma_{V_{e y}}^{2}+\lambda\right)\left(\sigma_{V_{e z}}^{2}+\lambda\right)}} \cdot \frac{1}{\sigma_{V_{e x, e y, e z}}^{2}+\lambda} \\
& \quad \cdot \exp \left[-\frac{1}{2}\left(\frac{V_{i x}^{2}}{\sigma_{V_{e x}}^{2}+\lambda}+\frac{V_{i y}^{2}}{\sigma_{V_{e y}}^{2}+\lambda}+\frac{V_{i z}^{2}}{\sigma_{V_{e z}}^{2}+\lambda}\right)\right] d \lambda,
\end{aligned}
$$

where $K=2 \sqrt{2 \pi} m_{e} r_{e}^{2} Z^{2} c^{4} L_{c} n_{e}(x, y, z)$ and $\sigma_{v_{e i}}$ is the rms value of electron velocity spread in direction $i$. As a function of $V_{i}$, the friction force is precalculated and stored in a table in the program. During tracking, the cooling kick on each particle is obtained by interpolating between values in the force table. In our case, the electron beam satisfies cylindrical symmetry, so a $2 \mathrm{D}$ force table is applied to speed up the calculation.

Another important effect is IBS, which is the main heating effect that cooling was designed to counteract. In the code, the Bjorken-Mtingwa IBS model is used, and the growth rates are calculated by the fast algorithm introduced by Nagaitsev [23-24]. The kinetic map on the macroparticles due to IBS is based on the Langevin equation:

$P_{i}(t+\Delta t)=P_{i}(t)-K_{i} P_{i}(t) \Delta t+\sqrt{\Delta t} \sum_{j=1}^{3} C_{i, j} \varsigma_{j}$,

where $\vec{P}=\left(\frac{1}{\gamma} \frac{\Delta p}{p}, x^{\prime}, y^{\prime}\right)^{T}$ is the particle moment vector in phase space, $K_{i}$ is the friction coefficient which is calculated from Zenkevich's model [25], $C_{i, j}$ is a function of the diffusion coefficient $D_{i, j}$, and $\varsigma_{j}$ is a random number with standard normal distribution. By neglecting the correlation between different dimensions, we obtain

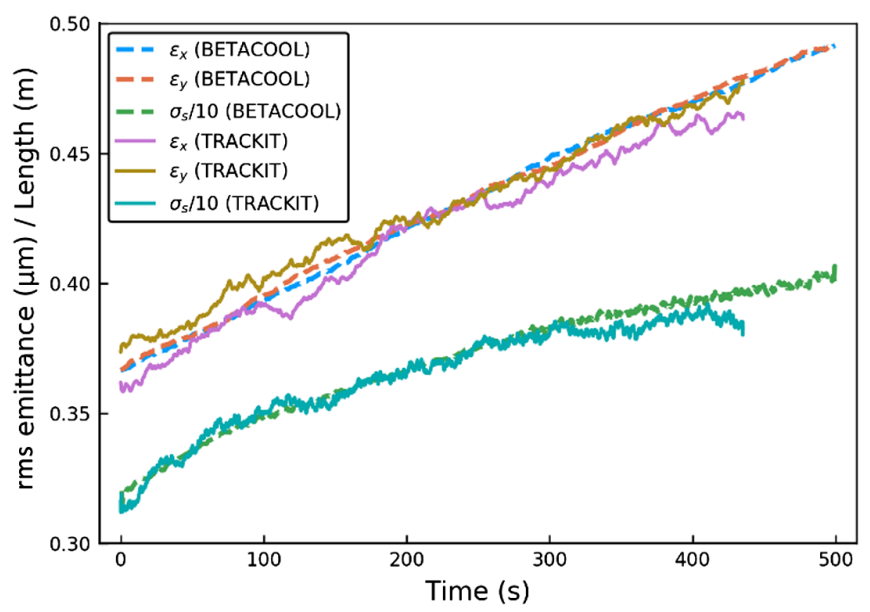

FIG. 3. Comparison of the growth of rms beam emittances $\left(\varepsilon_{x} / \varepsilon_{y}\right)$ and rms bunch length $\left(\sigma_{s}\right)$ caused by IBS for benchmarking with BETACOOL. 


$$
\sum_{j=1}^{3} C_{i, j} \varsigma_{j}=\varsigma_{i} C_{i, i}=\varsigma_{i} \sqrt{\left(2 K_{i}+\lambda_{i}\right) \cdot\left\langle P_{i}^{2}\right\rangle}
$$

where $\lambda_{i}=\frac{1}{\sigma_{p_{i}}^{2}} \frac{d \sigma_{p_{i}}^{2}}{d t}=\frac{1}{\epsilon_{i}} \frac{d \epsilon_{i}}{d t}$ is the IBS growth rate.

The IBS model in TRACKIT is benchmarked with BETACOOL. The result is shown in Fig. 3. In the calculation, the gold beam $\left({ }^{197} \mathrm{Au}^{79+}\right)$ is used with an initial rms emittance $\varepsilon_{x} / \varepsilon_{y}=0.37 / 0.37 \mu \mathrm{m}$ (coupled), rms bunch length $\sigma_{s}=3.2 \mathrm{~m}$, and rms momentum spread $d p / p=$ $4 \times 10^{-4}$. The $9 \mathrm{MHz}$ rf with $160 \mathrm{kV}$ total voltage is used. During tracking, linear coupled betatron motion is applied, and the IBS rate is calculated using the RHIC lattice at low energy $(\gamma=4.9)$. From Fig. 3, we see that the evolution of the beam emittance and bunch length from our code shows a good agreement with the BETACOOL results.

\section{B. SC effect on the electron beam}

Since there is no continuous solenoid field along the trajectory of the electron beam in the CSs, the SC effect of the electron beam itself will increase the electron velocity spread and, consequently, weaken the cooling force. In the particle frame, the length of the electron bunch in the CSs is much larger than its radius; therefore, the SC in the longitudinal direction can be ignored. In addition, the $\mathrm{SC}$ force of the ion beam on the electrons also needs to be considered [26]. Even though it can compensate the SC effect of electrons, the focusing strength depends on the ion charge density at the longitudinal position of the electron bunch. Because of that, the transverse distribution of each electron bunch is different and needs to be treated separately in the code.

In the simulation code, the parameters of the electron beam along the $\mathrm{CS}$ are calculated every $0.03 \mathrm{~m}$ based on the $\mathrm{SC}$ effect of electrons and ions. Assuming that the densities of the electron beam and the ion beam have a Gaussian distribution transversely, the SC force on electrons can be obtained by [27]

$F_{e, r}\left(r_{e e}, r_{e i}\right)=\frac{e\left[\frac{I_{e}}{r_{e e}}\left(1-e^{-\left(r_{e e}^{2} / 2 \sigma_{e}^{2}\right)}\right)-\frac{I_{i}}{r_{e i}}\left(1-e^{-\left(r_{e i}^{2} / 2 \sigma_{i}^{2}\right)}\right)\right]}{2 \pi \varepsilon_{0} \beta c \gamma^{2}}$,

where $r_{e e}$ and $r_{e i}$ are the distances between the electron and the center of the ion and electron beam, respectively, and $I$ is the beam current, which depends on the longitudinal position of the electrons, as shown in Fig. 2. Here, the electron beam and ion beam are considered as round beams transversely. The effects of solenoids and correctors are described by transfer matrices [28].

Based on the initial beam parameters, the properties of electron beam along the CS can be estimated. For example, Fig. 4 gives the evolution of the rms radius and rms velocity spread of the first 15 electron bunches along the CS of the yellow ring. The last 15 electron bunches are the same as
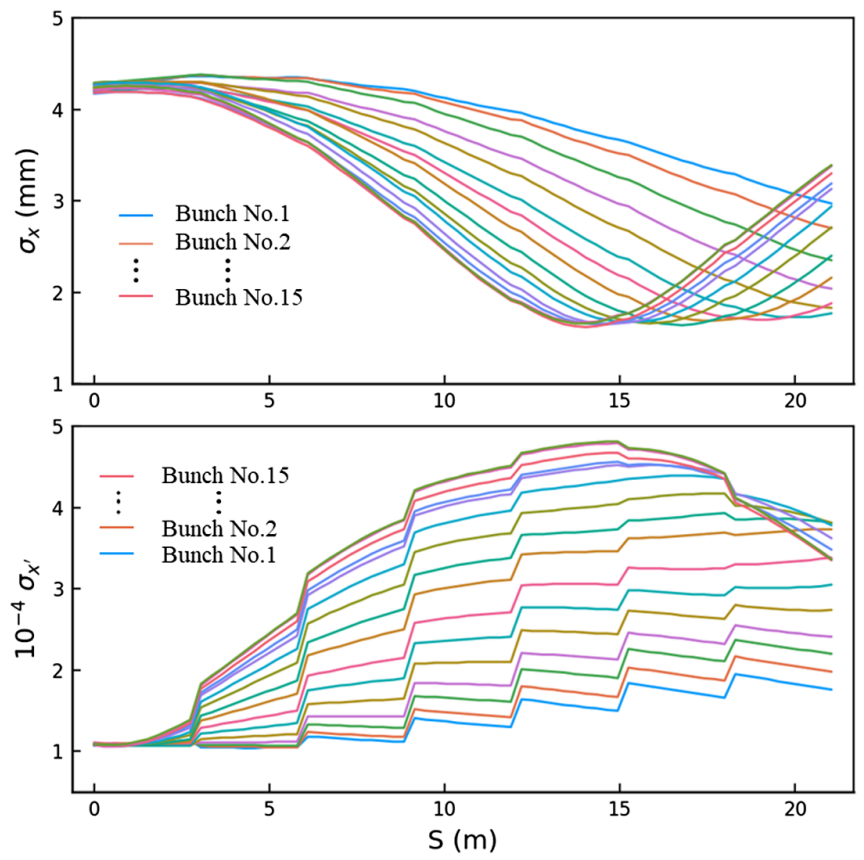

FIG. 4. The rms radius and rms momentum spread of the first 15 electron bunches along the $\mathrm{CS}$ of the yellow ring (the leaps on the momentum spread are due to the solenoid field).

the first 15 due to the symmetrical beam structure as shown in Fig. 2. The parameters in the simulation are listed in Table I. We can see that all electron bunches are focused, especially for the bunch at the center of the ion bunch.

In the experiment, yttrium-aluminum-garnet (YAG) screens in the CS measured the electron beam profile. For the beams with the same parameters listed in Table I, the measured electron beam profiles with and without the presence of an ion beam in the yellow ring are shown in Fig. 5. The profile is for the electron macrobunch which consists of 30 bunches. This YAG screen is located at the exit of the $180^{\circ}$ magnet that connects the CS of the yellow and blue rings. It clearly shows that the electron beam is focused by the ion beam. Comparing the experimental with the simulation result, it shows that the model is a reasonable approximation. However, the electron beam profile on the screen is asymmetric, which is due to the fact that the real electron beam is not an ideal round beam transversely.

TABLE I. Beam parameters at the start of the CS.

\begin{tabular}{lcc}
\hline \hline & Ion $\left({ }^{197} \mathrm{Au}^{79+}\right)$ & Electron \\
\hline Lorentz factor & $\gamma=4.1$ & $\gamma=4.1$ \\
Charge per bunch $(\mathrm{nC})$ & 8.2 & 0.05 \\
rms $\varepsilon_{x} / \varepsilon_{y}(\mu \mathrm{m})$ & $0.45 / 0.45$ & $0.45 / 0.45$ \\
Bunch length $(\mathrm{ns})$ & $12(\mathrm{rms})$ & 0.5 \\
$\beta$ function $(\mathrm{m})$ & 21 & 40 \\
$\alpha$ function & 0.0 & -0.2 \\
Solenoid field $(\mathrm{mT})$ & $\cdots$ & 3.5 \\
\hline \hline
\end{tabular}



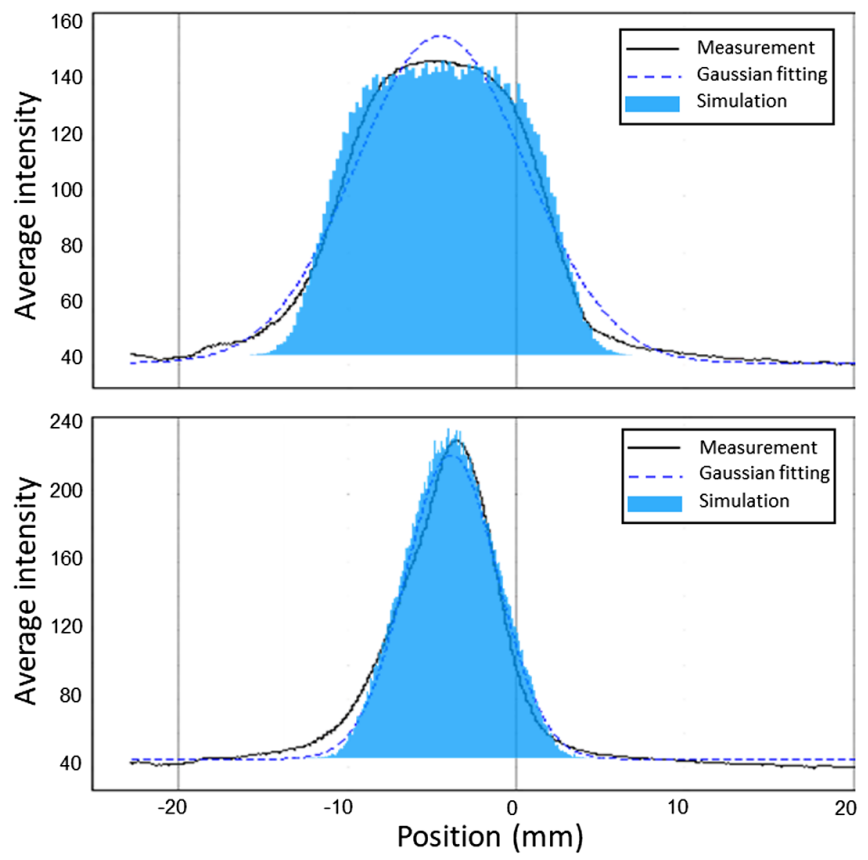

FIG. 5. Density distribution of the electron beam on a YAG screen and simulation result. Top: With no ion beam in the yellow ring. Bottom: With an ion beam in the yellow ring.

The position offset in the screen is caused by an angle error at the entrance of the $180^{\circ}$ magnet or the interference of the magnetic field from RHIC. These effects are not considered in the simulation, because they have little influence on the distribution of the electron beam.

Because of the SC effect from the ion beam, the distribution of each electron bunch is different and changes along the CS. Each electron bunch should be considered separately in the code, especially for the cooling module where cooling strongly depends on the electron beam distribution. In the code, the CS is divided into seven parts, in which the interactions between electrons and ions are considered independently. In each part, the cooling force for each electron bunch is precalculated based on its distribution. For example, Fig. 6 gives the simulation results of the cooling force for different electron bunches at the last part of the CS in the Yellow ring. The result is based on the parameters listed in Table I. Figure 6 clearly shows that cooling will be strongly affected by the SC effect. In addition, the angle error of the trajectory and position offset between ion beam and electron beam at each part of the CS are also included in the code.

\section{SC effect on the ion beam}

The SC effect of the electron beam on the ions is also included in the code. The transverse kick force from electron beam on the ions is obtained by

$$
F_{i, r}\left(r_{i e}\right)=-\frac{Z e I_{e}}{2 \pi \varepsilon_{0} \beta c \gamma^{2} r_{i e}}\left(1-e^{-\left(r_{i e}^{2} / 2 \sigma_{e}^{2}\right)}\right),
$$

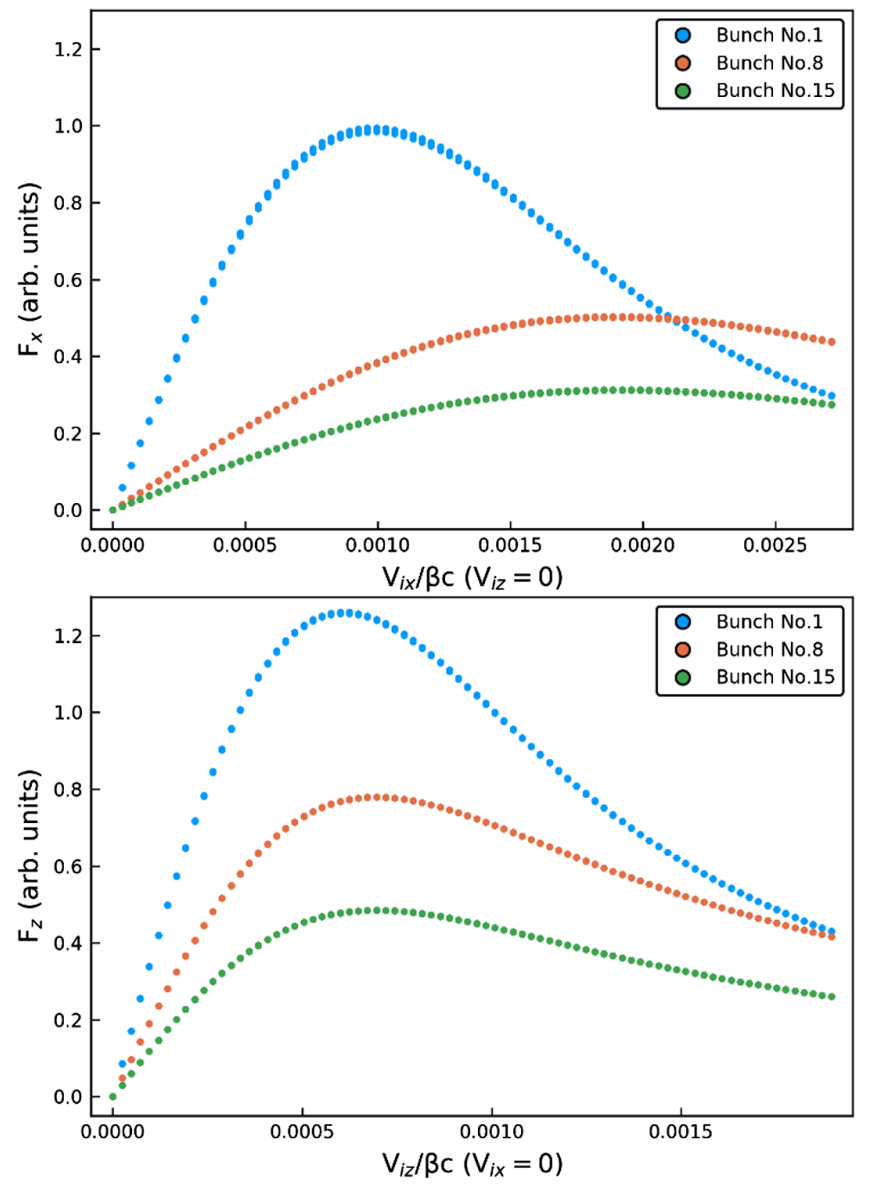

FIG. 6. Comparison of the cooling force for the electron bunches No. 1, 8, and 15 at the end of the CS of the yellow ring. Top: Transverse force. Bottom: Longitudinal force.

where $Z$ is the charge number of the ion. In the longitudinal direction, the electric field from the electron beam is given by

$$
E_{z}(z)=-\frac{g}{4 \pi \varepsilon_{0} \beta c \gamma^{2}} \frac{d I_{e}(z)}{d z}
$$

where $g=1+2 \ln (b / a)$ is the geometric factor and $a$ and $b$ are the radius of the beam and beam pipe, respectively. In our case, the electron bunch in the CS has a uniform distribution in the longitudinal direction. As a result, the longitudinal electric field exists only at the edges of the electron bunches. In the code, the effect of the electric field on the ion particles is treated as an accelerating or decelerating voltage. Ignoring the small velocity difference, the ions and electrons will propagate through the CS without relative position changes. So, the accelerating or decelerating voltage is given by $V_{z}(z)=L_{\mathrm{cool}} E_{z}(z)$, where $L_{\text {cool }}$ is the length of the CS [29].

According to the model introduced in Ref. [15], the transverse SC kicks from the electrons on the ions will excite synchrobetatron resonances, which cause betatron 

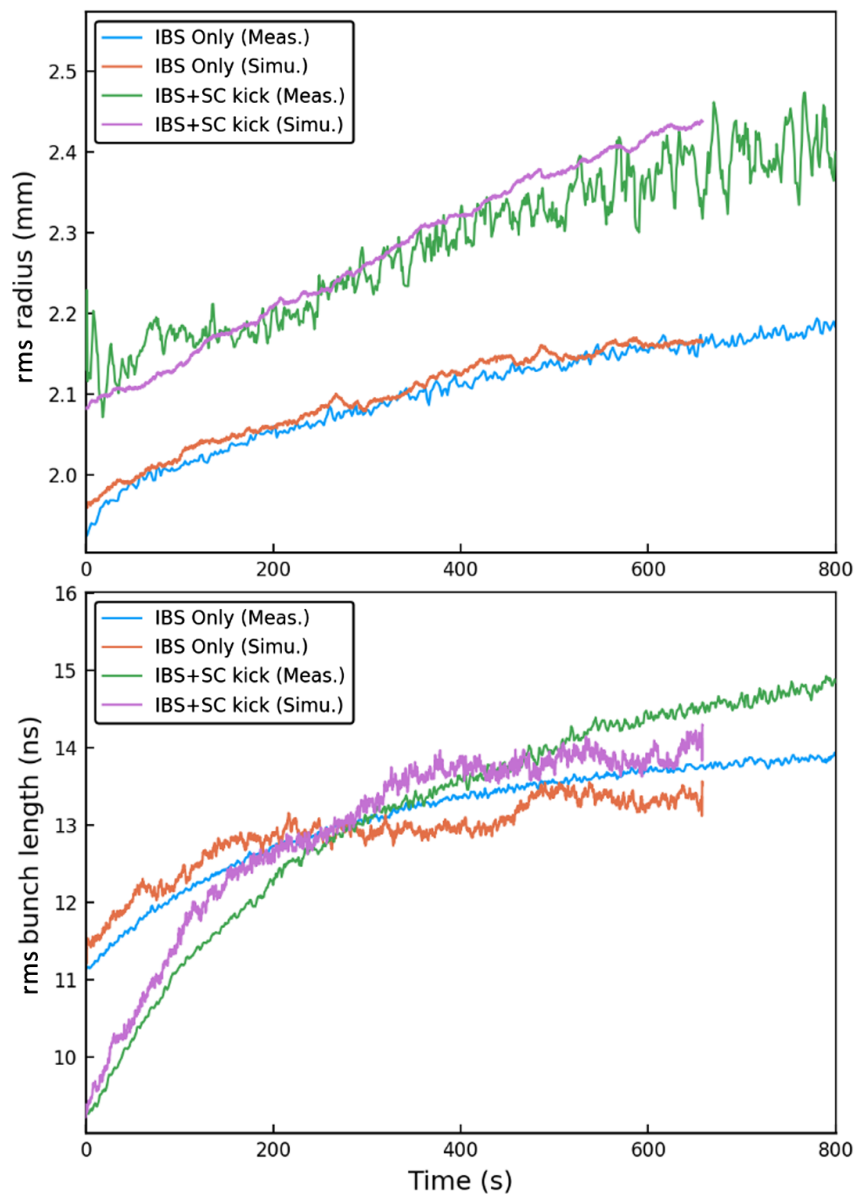

FIG. 7. Measurement and simulation results for the heating effect of the ion beam caused by IBS and the SC from the electron beam.

amplitude growth. Because the space-charge kick from the electrons is very small, one would expect these resonances to be negligible. However, due to the longitudinal IBS of the ions, individual particles will wander back and forth through these resonances. As a result, there is always a fraction of the ions affected by the resonance, resulting in emittance growth, which we refer to here as heating. Other explanations of the observed emittance growth due to the electron bunches are possible, but this model appears to be in agreement with our observations. In the absence of longitudinal IBS in simulations, no emittance growth is observed. When IBS is introduced, the observed emittance growth in simulations agrees with the measurements. In order to correctly account for this heating effect, the oneturn step tracking is required in the simulation. Because the CS is separated into several parts, the SC kicks on the ions are calculated and applied based on the average distribution of the electron beam in each part.

The emittance growth of the ion beam caused by the SC of electron bunches was experimentally measured. In the experiment, the electron bunches are propagated through the CS, and the cooling effect is switched off by mismatching the electron energy with the ions. As a result, the
TABLE II. Beam parameters for the heating study.

\begin{tabular}{lcc}
\hline \hline & Ion $\left({ }^{197} \mathrm{Au}^{79+}\right)$ & Electron \\
\hline Lorentz factor & $\gamma=4.9$ & $\gamma=4.9$ \\
Charge per bunch $(\mathrm{nC})$ & 8.8 & 0.06 \\
rms $\varepsilon_{x} / \varepsilon_{y}(\mu \mathrm{m})$ & $0.38 / 0.38$ & $0.5 / 0.5$ \\
Bunch length (ns) & $9(\mathrm{rms})$ & 0.5 \\
Rise/fall time (ps) & $\ldots$ & 20 \\
$d p / p$ & $3.5 \times 10^{-4}$ & $5 \times 10^{-4}$ \\
$\beta$ function @ H-jet (m) & 11.5 & $\ldots$ \\
$\beta$ function @ CS (m) & 21 & 25 \\
$\alpha$ function @ CS & 0.0 & -0.3 \\
Solenoid field (mT) & $\ldots$ & $\sim 3.2$ \\
rf voltage $(\mathrm{kV})$ & 160 & $\ldots$ \\
\hline \hline
\end{tabular}

ion beam dynamics depends on only the IBS effect and the $\mathrm{SC}$ from the electron beam. For comparison, the experiment with the electron beam off (IBS only) in RHIC was also performed. The beam parameters for simulation and experiment are listed in Table II, and the measurement results are shown in Fig. 7. The ion beam radius and bunch
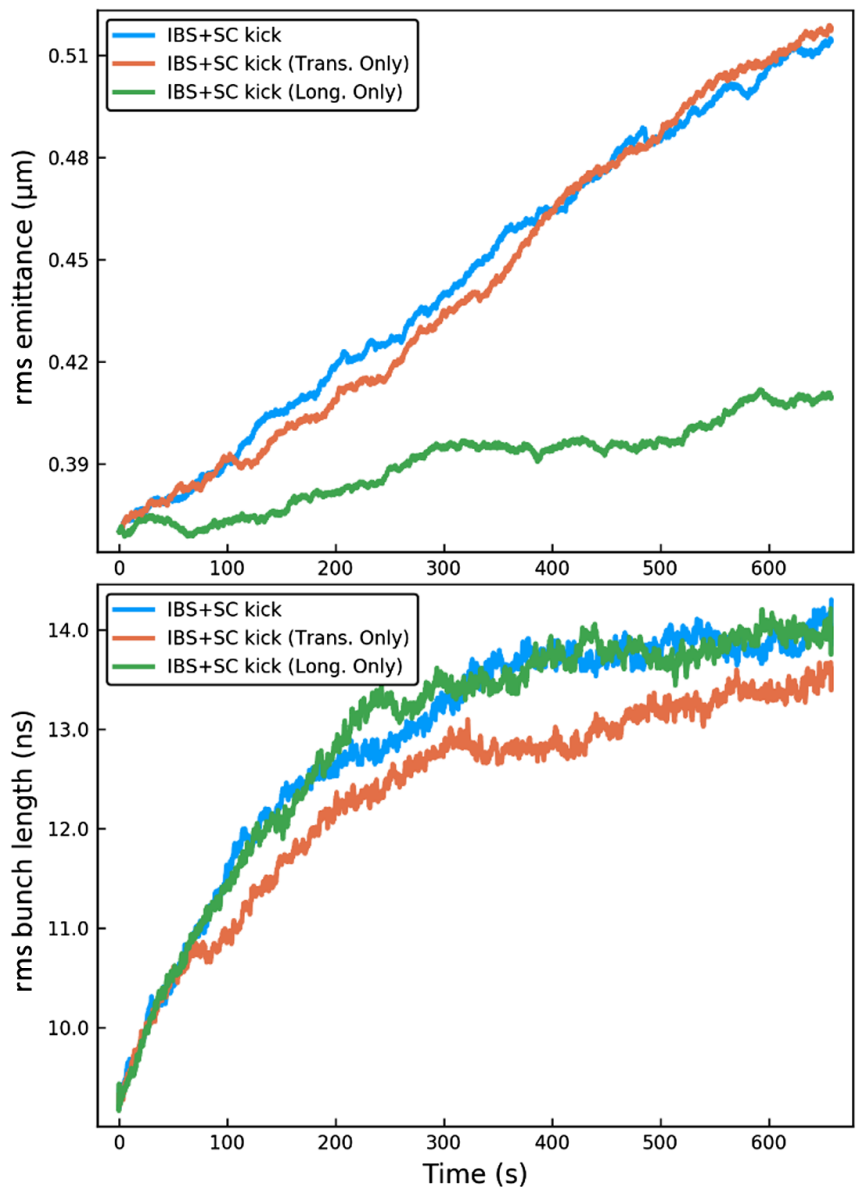

FIG. 8. Comparison of the effects of transverse and longitudinal SC kicks on the ion beam emittance and bunch length in simulations. 
length are measured by the H-jet polarimeter [30] and wallcurrent monitor, respectively. The simulation results are consistent with the measurement. These results indicate that the SC kicks of the electron beam increase the emittance growth rates of the ion beam, both transversely and longitudinally. The electron beam causes not only a cooling effect on the ions, but also a strong heating effect.

Below, we explore in detail effects of the transverse and longitudinal SC kicks from the electrons on the emittance growth of the ion beam. In the simulations shown in Fig. 8, the SC kicks in the transverse and longitudinal directions are considered independently. Both the SC effect of the electron beam and the IBS effect are included while the cooling is turned off. It shows that the transverse SC kick from electrons is the main source causing the beam emittance growth, which is much stronger than the IBS effect. The longitudinal SC kick has a small influence on the longitudinal dynamics compared to the effects of IBS. Based on Eq. (7), the accelerating or decelerating voltage from the electron beam is $4.0-6.0 \mathrm{kV}$, which is far less than the rf voltage $(160 \mathrm{kV})$. As a result, the longitudinal beam dynamics is mainly determined by the IBS effect.

\section{COOLING PROCESS}

LEReC has successfully demonstrated cooling of a gold beam in RHIC in 2019. A typical measurement of the cooling process in both the transverse and longitudinal directions is shown in Fig. 9. The initial parameters of the ion and electron beams are listed in Table III. The simulation results are also presented, in which all effects and various errors are considered. The errors come from the alignment error between the ion and electron beams. Even though several correctors and BPMs are installed in the CS to optimize and measure the electron beam trajectory, the position offset and the angle between the ion and electron beam trajectories are unavoidable. In the simulation, the trajectory of the ion beam is always assumed to be in the center of the beam pipe, and the two alignment errors are included with a constant position offset and a positive and negative staggered angle arrangement along the CS, which is close to the real condition in the experiment. As shown in Fig. 9, the alignment errors have a strong influence on the cooling process, especially in the transverse direction. Comparing the simulation results with the measurement, we estimate that the position offset of electron beam in the experiment is in the range of 0-2 $\mathrm{mm}$, and the angle between the electron and ion beam is about $50-100 \mu \mathrm{rad}$. In order to improve the cooling performance, these errors need to be restricted to a very small range.

In the simulation, the particle loss is accounted for by comparing the bunch particles with the rf bucket and the transverse aperture. As discussed before, the synchrobetatron resonances caused by the electron beam are one of the reasons for the beam loss. The beam lifetime at such low
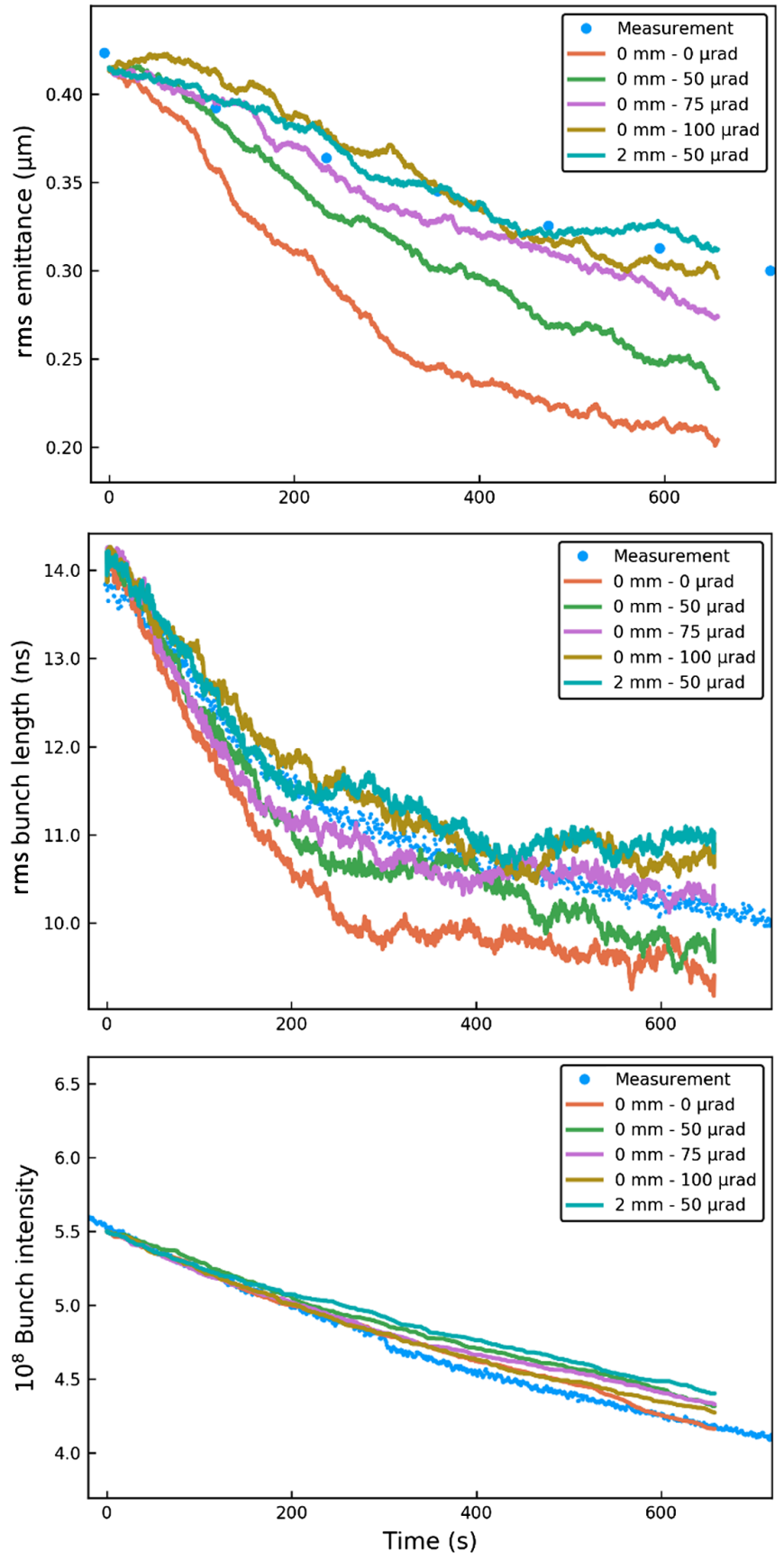

FIG. 9. Measurement and simulation results of the cooling process.

energy is also limited by the strong SC effect of the ion beam, residual gas scattering, the nonlinear magnetic field error stemming from persistent currents in the superconducting magnets, and the beam-beam effect in collisions [31]. These effects are not considered in the simulation. In order to match the decay of the beam intensity in the experiment, we set a dynamic aperture during particle tracking. With a 4-sigma aperture, the simulation results are in good agreement with the measurement, as shown in Fig. 9. 
TABLE III. Initial beam parameters in the experiment.

\begin{tabular}{lcc}
\hline \hline & Ion $\left({ }^{197} \mathrm{Au}^{79+}\right)$ & Electron \\
\hline Lorentz factor & $\gamma=4.1$ & $\gamma=4.1$ \\
Charge per bunch $(\mathrm{nC})$ & 7 & 0.05 \\
rms $\varepsilon_{x} / \varepsilon_{y}(\mu \mathrm{m})$ & $0.42 / 0.42$ & $0.45 / 0.45$ \\
Bunch length (ns) & $14(\mathrm{rms})$ & 0.4 \\
$d p / p$ & $4.6 \times 10^{-4}$ & $5 \times 10^{-4}$ \\
Rise/fall time (ps) & $\ldots$ & 20 \\
Tunes & $29.23 / 28.22$ & $\ldots$ \\
$\beta$ function @ CS (m) & 21 & 25 \\
$\alpha$ function @ CS & 0.0 & -0.3 \\
Solenoid field (mT) & $\ldots$ & $\sim 3.7$ \\
rf voltage $(\mathrm{kV})$ & 160 & $\ldots$ \\
Revolution frequency $(\mathrm{MHz})$ & 9.0 & 9.0 \\
\hline \hline
\end{tabular}

In addition, the charge of the electron bunch is an important factor which affects the cooling process. Based on the simulation code, we investigated the dependency of the cooling rate on the electron beam charge. Based
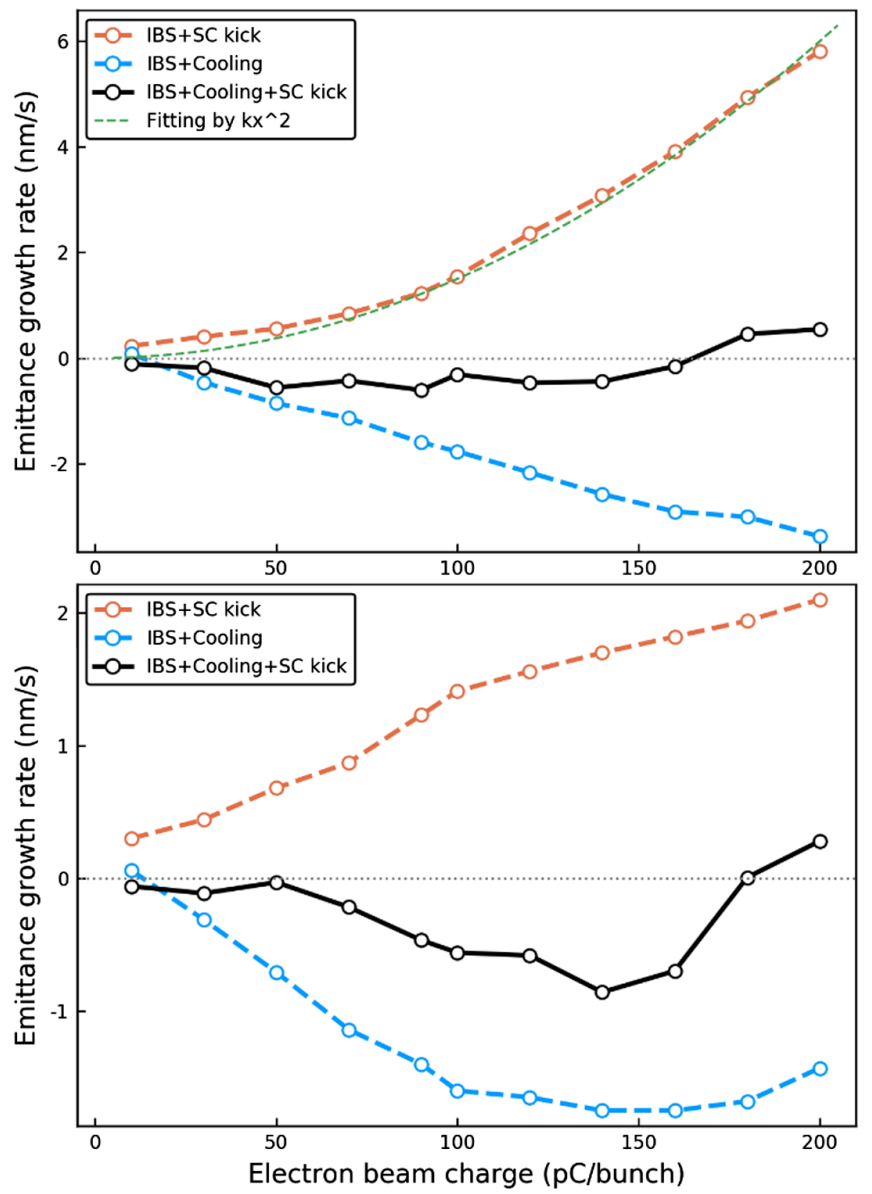

FIG. 10. Dependency of the emittance growth rate on the electron beam charge in simulation with (bottom) and without (top) considering the variable profile of the electron beam along the CS due to the SC effect. (Alignment errors, $0 \mathrm{~mm}-75 \mu \mathrm{rad}$.) on the analytical solution in Ref. [32], it is estimated that the emittance growth rate is proportional to the square of the electron bunch charge. But the changes of electron beam profile along the CS were not included in the physical model. In our simulation, we assume the same initial parameters of electron beam for different charges, and the cooling and heating effects are considered independently. Based on the evolution of the beam emittance in the first $50 \mathrm{~s}$, the dependence of the emittance growth rates on the electron bunch charge with and without the consideration of the variation of the electron beam along the CS due to SC effect are shown in Fig. 10. In the first case without considering the electron SC effect, the distributions of the electron beams are the same for different charges. It demonstrates that the growth rate is proportional to the square of the electron bunch charge. The cooling rate is proportional to the charge, which agrees with Eq. (1). However, due to the SC effect, the electron beam distribution along the CS strongly depends on the charge density. As shown in Fig. 10, in the more realistic case, both the growth rate and the cooling rate are reduced for high-charge electron beams, which is due to the large velocity spread and large beam size caused by the strong SC effect. This suggests that the best choice of electron beam charge is about $130 \mathrm{pC}$, close to the LEReC design value.

These results indicate that a very strict alignment between ion and electron beams is needed. To improve the cooling performance in the experiment, it requires not only to have a high-quality electron beam, but also to have a very stable and accurate measurement of the beam trajectory. If a similar emittance can be obtained for different electron beam charges, the charge of $130 \mathrm{pC}$ would provide the best performance of cooling in operation.

Moreover, because of the synchrobetatron resonance, which is the main heating effect during cooling, the synchrotron and betatron tunes need to be optimized for

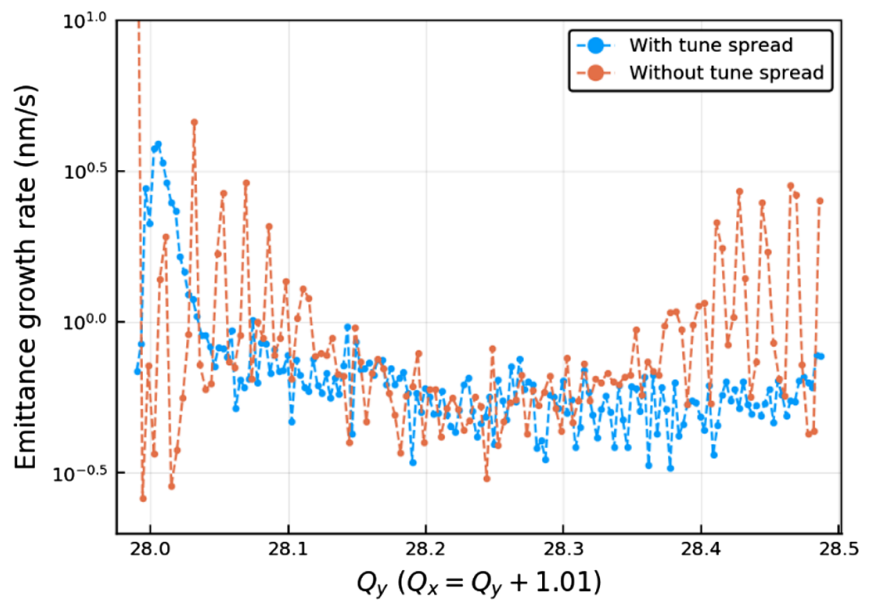

FIG. 11. Dependency of the emittance growth rate on the betatron tune with and without considering the tune spread in the simulation. 
good cooling performance. Based on the simulation tool, the dependency of the emittance growth rate on the betatron tune is investigated. The simulation results with and without considering the tune spread are shown in Fig. 11. In the simulation, the tune spread is about 0.1 , which comes from the SC effect of the ion beam. Figure 11 shows that the heating effect becomes stronger when the tune is closer to integer and half integer, and it is very sensitive to the absolute tune if there is no tune spread. It indicates that the tune should be set in the range of 0.2-0.4 to minimize the heating effect. But the best choice for the betatron tune should be based on the operation, because the ion beam lifetime and stability are also determined by the tune. This is consistent with experimental observations. Tunes between 0.1 and 0.5 were tested and the best overall performance found near 0.22 .

\section{SUMMARY}

We reported on experimental and simulation results of the world's first electron cooler based on rf-accelerated electron bunches. In the simulation code, the physical models for intrabeam scattering and space-charge effects are benchmarked against other codes and experimental data. The simulated cooling process replicates the experimental result. It demonstrated that the SC effects of the electron and ion beams not only have a strong influence on the cooling force, but also introduce an extra heating effect. In order to optimize the cooling performance, the alignment of electron beam trajectory with the ion beam, the electron beam charge, and the betatron tune are the important factors to be considered in the operation.

\section{ACKNOWLEDGMENTS}

The authors thank Y. Luo and H. Huang for many useful suggestions and discussions on the simulation and benchmarking. This work was supported by the U.S. Department of Energy under Contract No. DE-SC0012704.

[1] G. I. Budker, An effective method of damping particle oscillations in proton and antiproton storage rings, Sov. At. Energy 22, 438 (1967).

[2] I. N. Meshkov, Electron cooling: Status and perspectives, Phys. Part. Nucl. 25, 631 (1994); https://cds.cern.ch/ record/398450/files/p26.pdf.

[3] H. Poth, Applications of electron cooling in atomic, nuclear and high-energy physics, Nature (London) 345, 399 (1990).

[4] M. Chanel, Leir: The low energy ion ring at CERN, Nucl. Instrum. Methods Phys. Res., Sect. A 532, 137 (2004).

[5] P. Kienle, The heavy ion cooler and synchrotron ring at GSI, Nucl. Phys. A447, 419 (1986).

[6] D. Prasuhn, J. Dietrich, R. Maier, R. Stassen, H. J. Stein, and H. Stockhorst, Electron and stochastic cooling at
COSY, Nucl. Instrum. Methods Phys. Res., Sect. A 441, 167 (2000).

[7] J. W. Xia et al., The heavy ion cooler-storage-ring project (HIRFL-CSR) at Lanzhou, Nucl. Instrum. Methods Phys. Res., Sect. A 488, 11 (2002).

[8] S. Nagaitsev et al., Experimental Demonstration of Relativistic Electron Cooling, Phys. Rev. Lett. 96, 044801 (2006).

[9] C. Montag, eRHIC design overview, in Proceedings of the 10th International Particle Accelerator Conference (IPAC'19), Melbourne, Australia, 2019 (JACoW, Geneva, 2019), MOZZPLS1, http://accelconf.web.cern.ch/ipac2019/ papers/mozzpls1.pdf.

[10] Y. Zhang, JLEIC: A high luminosity polarized electron-ion collider at Jefferson Lab, in Proceedings of the 10th International Particle Accelerator Conference (IPAC'19), Melbourne, Australia, 2019 (JACoW, Geneva, 2019), TUPRB112, http://accelconf.web.cern.ch/ipac2019/doi/ JACoW-IPAC2019-TUPRB112.html.

[11] A. V. Fedotov et al., Accelerator physics design requirements and challenges of RF based electron cooler LEReC, in Proceedings of the North American Particle Accelerator Conference (NAPAC'16), Chicago, IL, USA, 2016 (JACoW, Geneva, 2017), WEA4CO05, http://accelconf.web .cern.ch/napac2016/papers/wea4co05.pdf.

[12] C. Liu et al., Improving the luminosity for beam energy scan II at RHIC, in Proceedings of the 10th International Particle Accelerator Conference (IPAC'19), Melbourne, Australia, 2019 (JACoW, Geneva, 2019), MOPMP044, http://accelconf.web.cern.ch/ipac2019/papers/mopmp044 .pdf.

[13] A. V. Fedotov et al., Experimental Demonstration of Hadron Beam Cooling Using Radio-Frequency Accelerated Electron Bunches, Phys. Rev. Lett. 124, 084801 (2020).

[14] D. Kayran et al., High-brightness electron beams for linacbased bunched beam electron cooling, Phys. Rev. Accel. Beams 23, 021003 (2020).

[15] M. Blaskiewicz, Emittance growth from modulated focusing in bunched beam cooling, in Proceedings of the North American Particle Accelerator Conference (NAPAC'16), Chicago, IL, USA, 2016 (JACoW, Geneva, 2017), WEA3IO01, http://accelconf.web.cern.ch/napac2016/ papers/wea3io01.pdf.

[16] S. Seletskiy et al., Accurate setting of electron energy for demonstration of first hadron beam cooling with rf-accelerated electron bunches, Phys. Rev. Accel. Beams 22, 111004 (2019).

[17] M. Blaskiewicz et al., Emittance growth from modulated focusing and bunched electron beam cooling, in Proceedings of COOL2015, Newport News, VA, USA, 2015 (JACoW, Geneva, 2016), WEXAUD02, http://accelconf .web.cern.ch/cool2015/papers/wexaud02.pdf.

[18] X. Gu et al., Stable operation of a high-voltage highcurrent dc photoemission gun for the bunched beam electron cooler in RHIC, Phys. Rev. Accel. Beams 23, 013401 (2020).

[19] B. Xiao et al., Design and test of $704 \mathrm{MHz}$ and $2.1 \mathrm{GHz}$ normal conducting cavities for low energy RHIC electron cooler, Phys. Rev. Accel. Beams 22, 030101 (2019). 
[20] A. O. Sidorin, I. N. Meshkov, I. A. Seleznev, A. V. Smirnov, E. M. Syresin, and G. V. Trubnikov, BETACOOL program for simulation of beam dynamics in storage rings, Nucl. Instrum. Methods Phys. Res., Sect. A 558, 325 (2006).

[21] Y. S. Derbenev and A. N. Skrinsky, The kinetics of electron cooling of beams on heavy particle storage rings, Part. Accel. 8, 1 (1977); https://cds.cern.ch/record/923404/files/ p1.pdf.

[22] T. Schramm, Realistic elliptical potential wells for gravitational lens models, Astron. Astrophys. 231, 19 (1990); T. Schrammhttp://adsabs.harvard.edu/full/1990A $\% 26$ A...231...19S.

[23] J. D. Bjorken and S. K. Mtingwa, Intrabeam scattering, Part. Accel. 13, 115 (1982); https://cds.cern.ch/record/ 140304/files/p115.pdf; Report No. FERMILAB-PUB-8247-THY.

[24] S. Nagaitsev, Intrabeam scattering formulas for fast numerical evaluation, Phys. Rev. Accel. Beams 8, 064403 (2005).

[25] P. Zenkevich, O. Boine-Frankenheim, and A. Bolshakov, A new algorithm for the kinetic analysis of intra-beam scattering in storage rings, Nucl. Instrum. Methods Phys. Res., Sect. A 561, 284 (2006).
[26] S. Seletskiy, M. Blaskiewicz, A. Fedotov, D. Kayran, and J. Kewisch, Effect of beam-beam kick on electron beam quality in first bunched electron cooler, J. Phys. Conf. Ser. 1350, 012134 (2019).

[27] K. Schindl, Space charge, in Beam Measurement (World Scientific, Singapore, 1999), pp. 127-151.

[28] S. Y. Lee, Accelerator Physics (World Scientific, Singapore, 2018).

[29] H. Zhao et al., Simulation of ion beam cooling with a pulsed electron beam, Nucl. Instrum. Methods Phys. Res., Sect. A 902, 219 (2018).

[30] A. Zelenski et al., Absolute polarized H-jet polarimeter development, for RHIC, Nucl. Instrum. Methods Phys. Res., Sect. A 536, 248 (2005).

[31] A. V. Fedotov et al., Beam lifetime and limitations during low-energy RHIC operation, in Proceedings of the 24th Particle Accelerator Conference, PAC-2011, New York, 2011 (IEEE, New York, 2011), THP081, https://accelconf .web.cern.ch/accelconf/PAC2011/papers/thp081.pdf.

[32] S. Seletskiy, Electron-beam heating in rf-based electron cooler (to be published). 